\title{
Nonlinear Analysis of Return Time Series Model by Oriented Percolation Dynamic System
}

\author{
Anqi Pei and Jun Wang \\ Institute of Financial Mathematics and Financial Engineering, School of Science, Beijing Jiaotong University, \\ Beijing 100044, China
}

Correspondence should be addressed to Jun Wang; wangjun@center.njtu.edu.cn

Received 13 June 2013; Revised 18 September 2013; Accepted 18 September 2013

Academic Editor: Luca Guerrini

Copyright (c) 2013 A. Pei and J. Wang. This is an open access article distributed under the Creative Commons Attribution License, which permits unrestricted use, distribution, and reproduction in any medium, provided the original work is properly cited.

Fluctuation dynamics of financial price changes is developed and investigated by oriented percolation system; oriented percolation is percolation with a special direction along which the activity can only propagate one way but not the other. Then, nonlinear behaviors of distribution and leverage effect of return time series are studied for the proposed model and the real stock market by comparison. We also investigate the scaling behaviors of return intervals. And a scaling function of exponential parameter is introduced to analyze fluctuation behaviors of return intervals. The empirical research exhibits that, for proper parameters, the simulation data of the model can fit the real markets to a certain extent.

\section{Introduction}

Fluctuation behaviors of financial market indexes and returns have long been a focus of economic research. Some behaviors (or stylized facts) of market fluctuations are uncovered by high frequency financial time series, such as powerlaw tail distribution of returns, volatility clustering, and aggregational gaussianity and multifractality of volatility [112]. There has been considerable interest in application of statistical physics to financial market dynamics [13-22], since econometric modeling is vital in finance and in financial time series analysis. The modeling of dynamics of forwards prices is becoming a key problem in the risk management, physical assets valuation, derivatives pricing, and so forth. Any modeling, the creation of representations of reality, aiming at understanding price fluctuations needs to define a mechanism for the formation of the price, in an attempt to reproduce and explain this set of stylized facts [23-25]. Percolation, the popular model of statistical physics systems [26-30], has been employed to model and explore these empirical facts of price changes in financial markets $[3,13-$ 16]. For example, Stauffer and Aharony [14] developed a price model by lattice percolation, the local interaction or influence among traders in one stock market is modeled, and a cluster of percolation is applied to describe the cluster of traders sharing the same opinion about the market. Recently, statistical behaviors of waiting times between two successive price changes with high frequency financial data have been analyzed [12, 16, 22, 31]. Wang et al. [31] studied the return intervals between price volatilities which are above some threshold. They also showed that the probability density function (PDF) of return intervals follows a certain scaling function. In the present paper, the local interaction among traders is developed by the oriented percolation to reproduce the nature of stock price fluctuations. We assume that the dispersal of information in the stock market affects the investment decisions of market participants and contribute the stock price fluctuations to the traders' investment decisions toward the stock market. Then we study the powerlaw distributions and the leverage effects of returns for the proposed model and the real stock market indexes. Further, we investigate the probability density functions of return intervals series of the simulation data and the actual data. Then a scaling function of exponential parameter is introduced to analyze the corresponding scaling relationships, and this new approach may be useful to bring out some statistical 


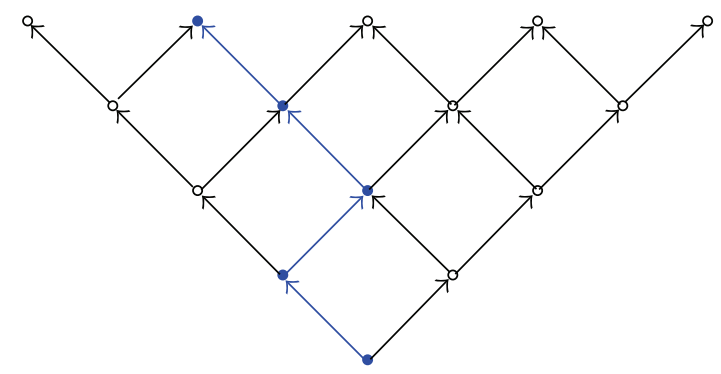

FIGURE 1: Schematic diagram of two-dimensional oriented percolation. The arrows indicate the direction of percolation.

properties for the return intervals. For the empirical research, the price data of Shanghai Stock Exchange Composite Index (SSE) and Shenzhen Stock Exchange Component Index (SZSE) are selected as the empirical data.

\section{Financial Time Series Model}

2.1. Brief Description of Oriented Percolation. Oriented percolation originally concerns a class of models that mimic filtering of fluids through porous materials along a given direction. In recent years, percolation theory, which describes the behavior of connected clusters in a random graph, as an extensive mathematical model of percolation, has emerged as a cornerstone of the disordered physical system theory and also has brought new understanding to a broad range of topics in society $[9,14,26-29]$. We consider a regular lattice, like a square lattice, and make it into a random network by randomly "occupying" bonds (edges) with a statistically independent probability $p$. Let $\mathscr{V}=\left\{(m, n) \in \mathbb{Z}^{2}: m+\right.$ $n$ is even, $m$ and $n$ are integers, and $n \geq 0\}$, and draw an oriented arc from each $(m, n) \in \mathscr{V}$ only to $(m+1, n+1)$ and to $(m-1, n+1)$, see Figure 1 . Each arc, also called a bond (edge), is independently open with probability $p(0 \leq p \leq 1)$ and closed with probability $1-p$. We have $x \mapsto y$ if there is an open path from $x$ to $y$; that is, there is a sequence $x=x_{0}, x_{1}, \ldots, x_{j}=y$ of points in $\mathscr{V}$ such that for each $k \leq j$, the arc from $x_{k-1}$ to $x_{k}$ is open. Considering the subgraph consisting of open paths in the lattice, the connected parts of subgraph constitute the open cluster. Let $D(x)=\{y$ : $x \mapsto y$ \} denote the open cluster containing $x$, and $|D(x)|$ is the number of vertices in $D(x)$, where $D(0)$ is the set of all the points included in an open cluster from the origin of coordinates. The infinite open cluster event is defined by

$$
\begin{aligned}
\Omega_{\infty} & =\{|D(0)|=\infty\} \\
& =\{\text { there is an infinite open path from }(0,0)\} .
\end{aligned}
$$

The state of the dynamic system from the original point at "time $n$ " is given by $\xi_{n}^{\{0\}}=\{m:(m, n) \in \mathscr{V}$ and $0 \mapsto(m, n)\}$. The most important feature of oriented percolation system is the critical point $p_{c}=\inf \left\{p: P\left(\xi_{n}^{\{0\}} \neq \emptyset\right.\right.$, for all $\left.n\right)>$ $0\}$, which is the probability that the infinite connectivity of percolation first occurs; that is, percolation networks exhibit the critical phenomena. When $p>p_{c}$, the percolation cluster tends to infinity, and also the percolation occurs. According to the percolation theory $[26,27]$, the critical value of $p_{c}$ can be estimated by $0.6298 \leq p_{c} \leq 0.84$. Let

$$
r_{n}^{\{0\}}=\sup \xi_{n}^{\{0\}}, \quad l_{n}^{\{0\}}=\inf \xi_{n}^{\{0\}},
$$

where we suppose that $\sup \emptyset=-\infty$ and inf $\emptyset=+\infty$. And $r_{n}^{\{0\}}$ and $l_{n}^{\{0\}}$ stand for the right edge process and the left edge process of $\xi_{n}^{\{0\}}$, respectively. Then we have the following results $[26,27]$. On $\Omega_{\infty}$, in the supercritical case $p>p_{c}$, we have

$$
\lim _{n \rightarrow \infty} \frac{r_{n}^{\{0\}}}{n}=\alpha(p), \quad \lim _{n \rightarrow \infty} \frac{l_{n}^{\{0\}}}{n}=-\alpha(p), \quad \text { a.s. }
$$

where $\alpha(p)>0$. In the subcritical case $p<p_{c}$, the process $\xi_{n}^{\{0\}}$ dies out exponentially fast. There is a constant $\gamma(p)>0$ so that

$$
P\left(\xi_{n}^{\{0\}} \neq \emptyset\right) \leq e^{-\gamma n}, \quad \frac{1}{n} \ln P\left(\xi_{n}^{\{0\}} \neq \emptyset\right) \longrightarrow \gamma \quad \text { as } n \longrightarrow \infty .
$$

In the present paper, the interaction among particles of oriented percolation is applied to describe the interaction among investors in a financial market, and the open probability $p$ of a bond is considered as the dispersal intensity of information.

2.2. Financial Price Model. Financial time series analysis is an active topic to understand the behavior of stock price fluctuation, which has long been a focus of economic research $[23,24]$. And econometric modeling is vital in finance and in financial time series analysis. In this section, the oriented percolation is applied to construct the financial price model, which is considered as the creation of representation of reality.

In the present paper, we assume that the price fluctuation results from the traders' investment attitudes toward the stock market. The open cluster in percolation theory is used to define the cluster of traders sharing the same opinion (or same information) on the market, and then the local interaction among traders in a stock market is modeled. The proposed financial model is constructed as follows. Consider a two-dimensional network, a $L \times L$ lattice, where $L$ is a positive integer. The node of the $L \times L$ lattice represents 


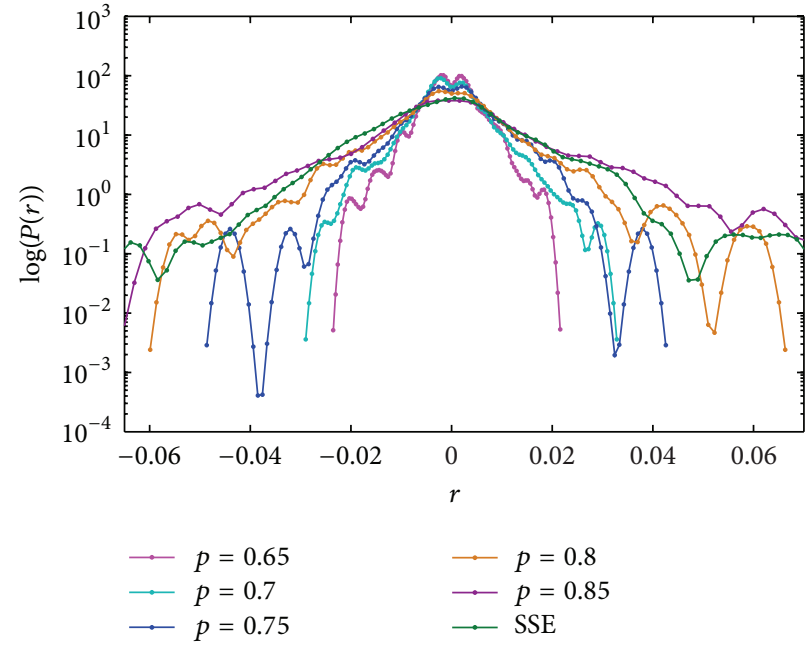

(a)

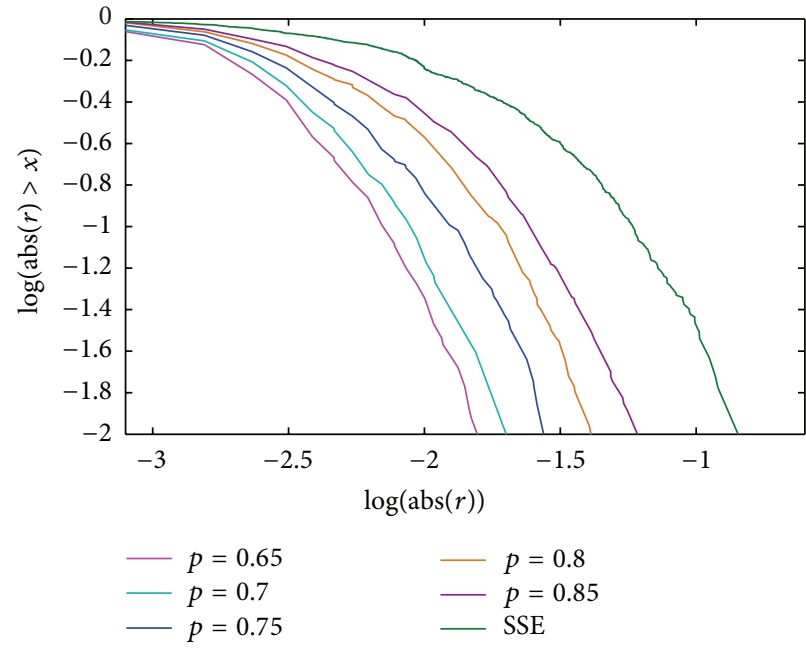

(b)

FIgure 2: (a) Probability density functions of returns for SSE index and the financial model with different values of $p$. (b) Corresponding power-law distributions of returns.

an agent in the real market, and the random percolation clusters are used as groups of traders. For each edge, it is independently designated as open with probability $p$ and closed with probability $1-p$. The trader can only transmit the information to his nearest two neighbors if the corresponding connected edge is open. Assume that each trader can trade the stock at every day $t$, and, at the beginning of trading day $t$, suppose that the investor at the origin of $D(0)$ receives some information. We define a random variable $\xi_{t}$ for this investor; suppose that this investor takes buying position $\left(\xi_{t}=+1\right)$, selling position $\left(\xi_{t}=-1\right)$, or neutral position $\left(\xi_{t}=\right.$ $0)$ with probability $p_{1}, p_{2}$, or $1-p_{1}-p_{2}$, respectively. Then this investor sends positive, negative, or neutral signal to his neighbors. Thus, the information can be transported just like the water filtering in percolation theory with the probability $p$. In the following, we let $p_{1}=p_{2}=0.5$, for a fixed $t$; the aggregate excess demand for the asset at time $t$ is defined by $\mathscr{A}(t)=\xi_{t} D(0) / L^{2}$, where $t \in\{1,2,3, \ldots, T\}$. From the above definitions and $[32,33]$, we define the formula of a discrete time stock price as

$$
\begin{aligned}
& S(t)=S(t-1) \exp \{\beta \mathscr{A}(t)\}, \\
& S(t)=S(0) \exp \left\{\beta \sum_{k=1}^{t} \mathscr{A}(k)\right\},
\end{aligned}
$$

where $\beta(>0)$ represents the depth parameter of the market and $S(0)$ is the initial stock price at time $t=0$. The formula of the stock logarithmic return $t$ to $t+1$ is given by

$$
r(t)=\ln S(t+1)-\ln S(t) .
$$

\section{Statistical Behaviors of Financial Time Series}

3.1. Distribution of Return Time Series. Empirical research shows that the fluctuation of returns is believed to follow a Gaussian distribution for long time range, but when being studied for short steps, especially for the tails, the probability density of returns would deviate from the Gaussian distribution while showing sharp peak and fat tail behaviors. Figure 2(a) exhibits the probability distributions of the simulation data (where we let the parameter $p=0.65,0.7,0.75$, 0.8 , and 0.85 ) and the actual data (SSE). Through calculation, we get the kurtosis values for the 6 data sets: $6.1877,7.5707$, $7.6983,7.7579,8.9145$, and 5.6012 , respectively. It is known that the kurtosis shows the centrality of data and the skewness shows the symmetry. While, for standard normal distribution the values are 3 and 0 , respectively. We find that the kurtosis values of the simulation data (which are all larger than 3 ) increase as the $p$ increases. Thus, the sharp peak of returns is obvious and the fat tail is also visible compared with the Gaussian distribution. Since the probability $p$ of the model relates to the spreading range of information, as $p$ increases, the market is going to be more active, resulting in large volatilities of stock prices. The cumulative distributions of returns in Figure 2(b) exhibit the power-law distributions, which is a universal property and largely observed in financial systems [23-25]. Empirical results show that the distribution of logarithmic returns follow a power-law distribution; that is, $P(r(t)>x) \sim x^{-\mu}$, where $r(t)$ is the returns of stock prices and the exponent $\mu$ is nearly 3 .

3.2. The Leverage Effect. The leverage effect, or volatility asymmetry, which corresponds to a negative correlation between past returns and future volatility [1, 4, 5], describes the volatility-return correlation. It is observed that the amplitude of relative price fluctuations of stock tends to increase when its price drops. According to this, a price drop increases the risk of a company to go bankrupt, so the stock will become more volatile $[2,10]$. Although various single correlation coefficients quantifying the leverage effect have been measured and discussed within GARCH-like models 


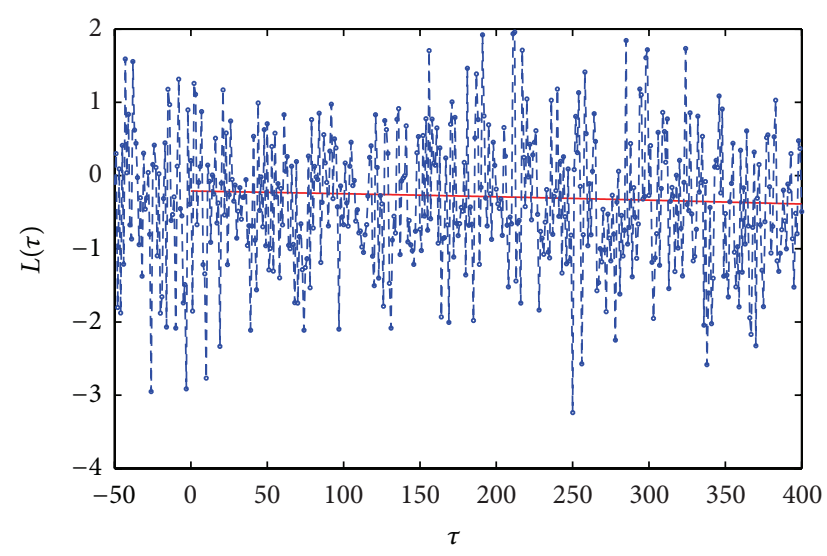

Coefficients (with 95\% confidence bounds): a: $-0.2879(-0.4404,-0.1354)$ b: $0.001268(-0.000775,0.0033)$

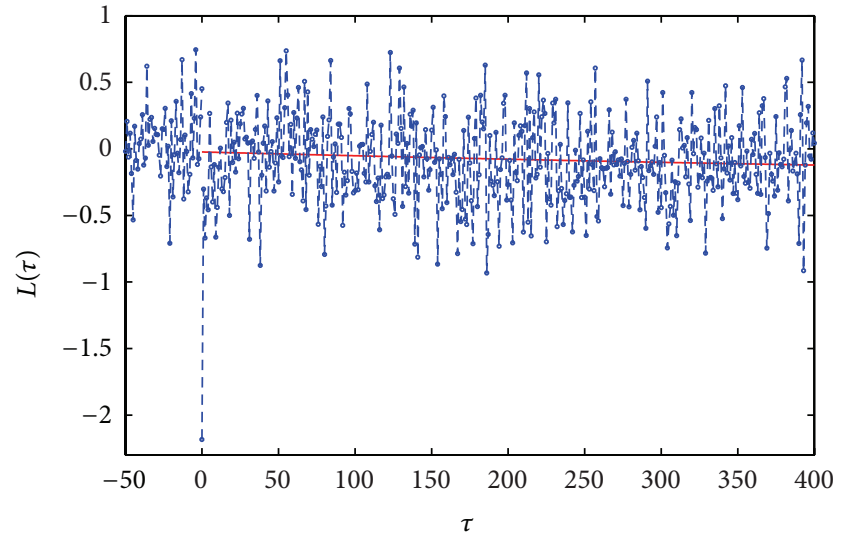

Coefficients (with 95\% confidence bounds): a: $-0.0916(-0.1545,-0.02866)$ b: $0.0004991(-0.002335,0.003333)$

(a)

(b)

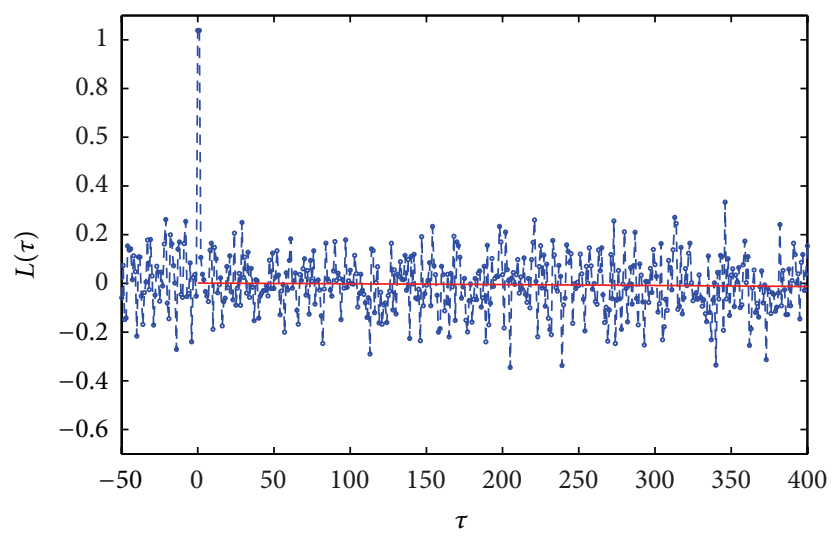

Coefficients (with 95\% confidence bounds):

$a:-0.00111(-0.00896,0.00674)$

b: $0.007611(-0.01309,0.02831)$

(c)

FIGURE 3: Volatility-return correlations of returns for the financial model with the dispersal intensity (a) $p=0.65$, (b) $p=0.75$, and (c) $p=0.85$.

[5], the full temporal structure of this correlation has not been widely investigated. So we explore a new point of view to study the volatility-return correlation for the return time series. The daily price change $\Delta S(t)$ is denoted by $\Delta S(t)=$ $S(t+1)-S(t)$, and the relative price change is given as $\Delta x(t)=\Delta S(t) / S(t)$; actually, $\Delta x(t)$ is equal to $r(t)$ which is defined in Section 2.2. The leverage correlation function which measures the correlation between the price change at time $t$ and a measure of the square volatility at time $t+\tau$ is given

$$
L(\tau)=\frac{1}{Z}\left\langle[\Delta x(t+\tau)]^{2} \Delta x(t)\right\rangle,
$$

where the bracket refers to a time average and the coefficient $Z$ is a normalization which is chosen as $Z=\left\langle[\Delta x(t)]^{2}\right\rangle^{2}$. We analyze the behaviors of the leverage correlation function for the simulation data with the parameters $p=0.65,0.75$, and
0.85 in Figure 3, while the time window $\tau$ ranging from -50 to 400. The corresponding leverage correlation function $L(\tau)$ for SSE and SZSE are displayed in Figure 4; here the daily closing price data from January 1991 to February 2012 are considered by this research.

The above empirical results tell us that the trend of $L(\tau)$ is various for negative values and positive values of $\tau$. We focus on positive values of $\tau$; as can be seen from these figures, $L(\tau)$ for all the data are significant and negative; price drops increase the subsequent volatility-this is the so-called leverage effect. Furthermore, this correlation function can be fitted by single exponentials:

$$
L_{\text {fit }}(\tau)=a \exp \{b \tau\} .
$$

Here, $|a|$ reflects the amplitude of volatility and $|1 / b|$ mirrors the decay time which presents an exponential decay. While it can be contrasted with the slow, power-law-like decay of 


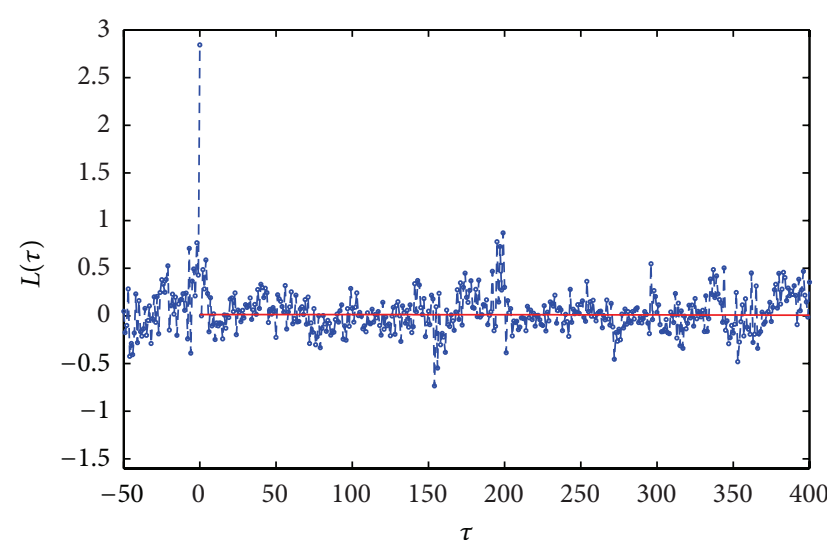

Coefficients (with $95 \%$ confidence bounds): a: $2.268 e-010(-3.286 e-009,3.739 e-009)$ b: $0.05265(0.01304,0.09225)$

(a)

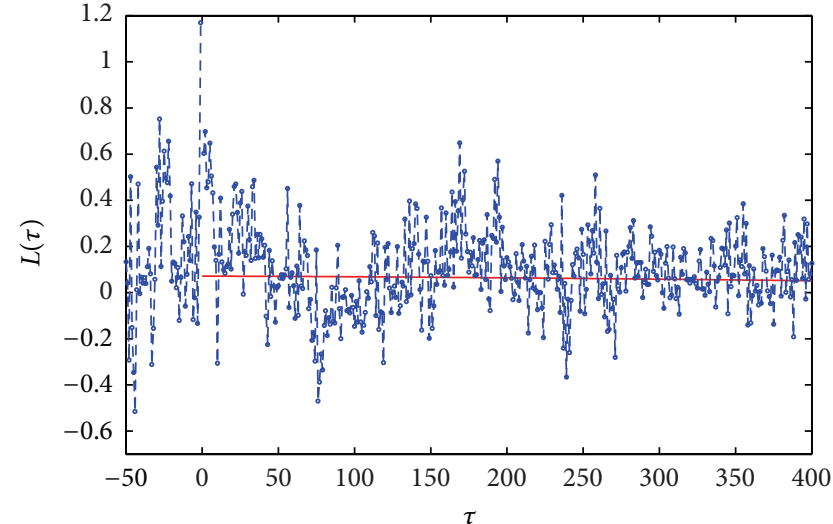

Coefficients (with $95 \%$ confidence bounds): a: $0.168(0.1149,0.2213)$ $b:-0.00292(-0.004848,-0.0009842)$

(b)

FIGURE 4: Volatility-return correlations of real returns for (a) SSE and (b) SZSE.

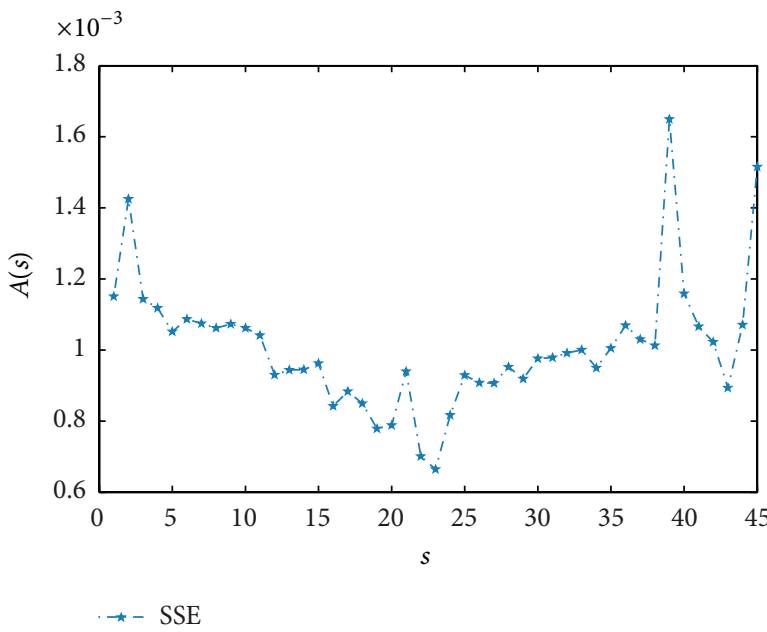

(a)

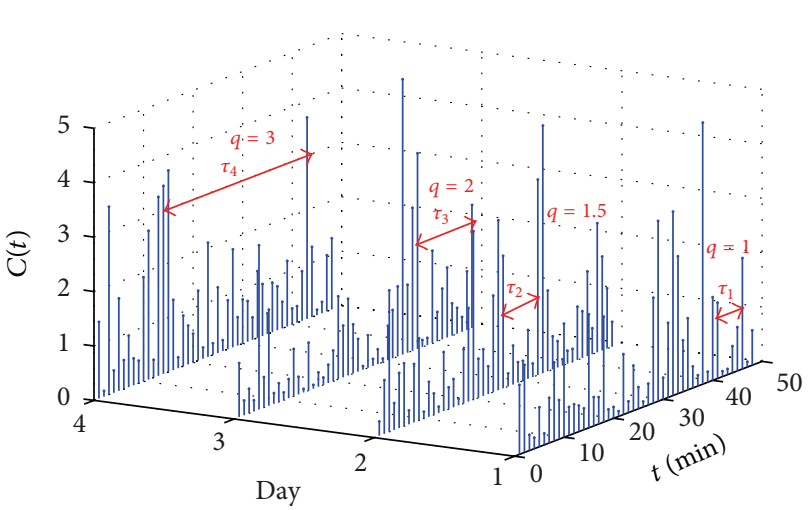

(b)

FIGURE 5: (a) The plot of 5 min interval intraday patterns for SSE. (b) The return intervals of SSE volatilities on different thresholds in four trading days.

the volatility correlation function, which can not be characterized by a unique decay time $[3,7,25]$, the insets of Figures 3 and 4 display the values of $a, b$ and the solid line shows the exponential fit.

\section{Systematic Comparisons for Return Interval Series}

In this section, we make systematic comparisons between the return intervals of the financial model and the actual data. The price series of SSE and SZSE from May 2001 to July 2005, which have $5 \mathrm{~min}$ interval length, are selected as the actual data. Then we study the statistical behaviors of the return intervals, especially for a new introduced exponent parameter in Section 4.3.
4.1. Description of Return Intervals. The characteristics of isolated high volatility events in price fluctuations and the distributions of return intervals between these events are of great interest in financial research. The intraday volatility return intervals reflect the time gaps between some fixed returns, which also show specific patterns because of different behaviors of traders at different periods in the trading day [31]. Let $A(s)$ denote the intraday pattern, which is the price change at a particular moment $s$ of the trading day averaged over all $N$ trading days and defined as

$$
A(s)=\sum_{k=1}^{N} \frac{r^{k}(s)}{N},
$$

where $r^{k}(s)$ is the logarithmic return at time $s$ of day $k$. Figure 5(a) is the plot of the intraday pattern $A(s)$ of SSE 


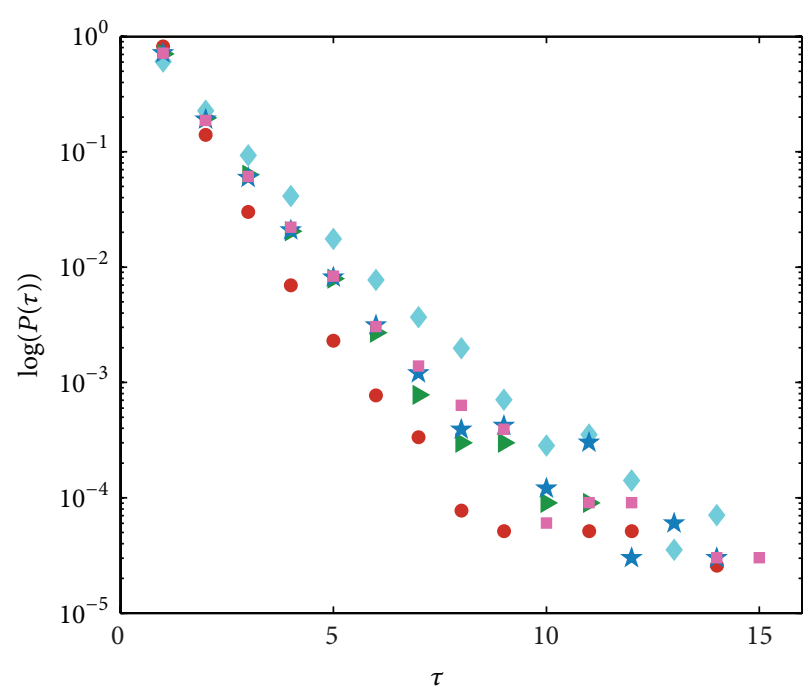

(a)

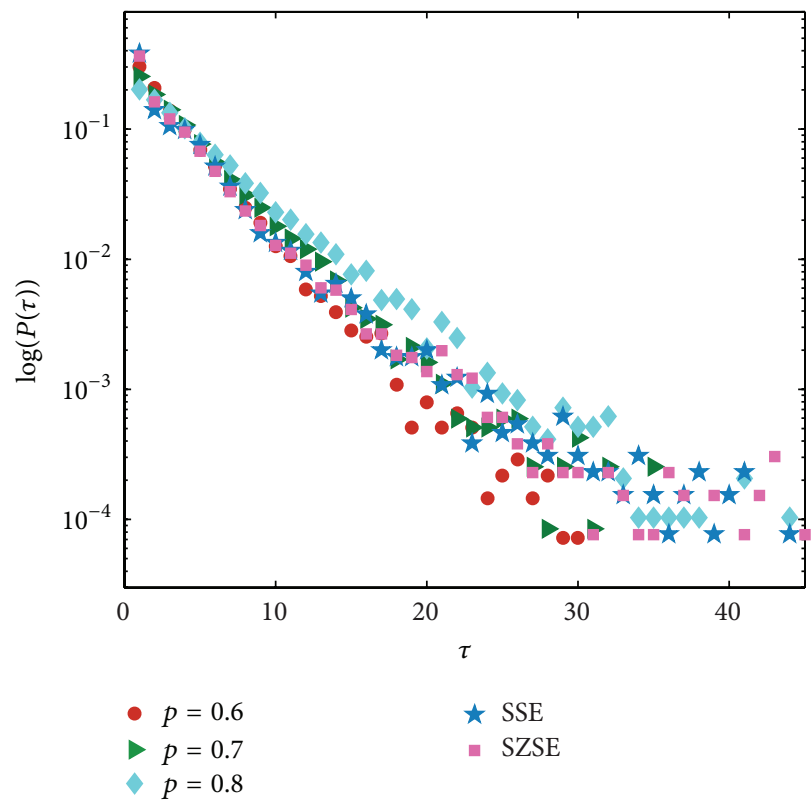

(c)

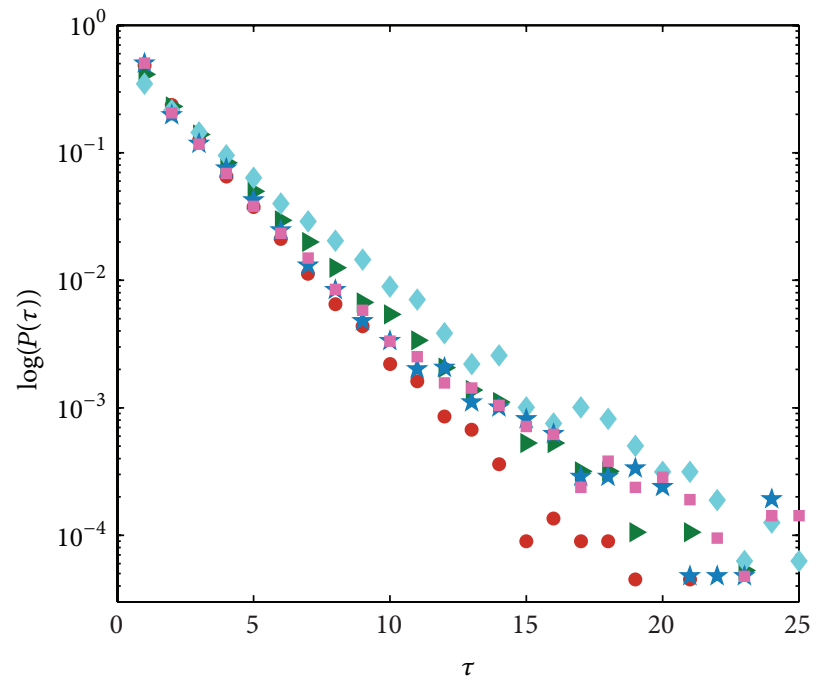

(b)

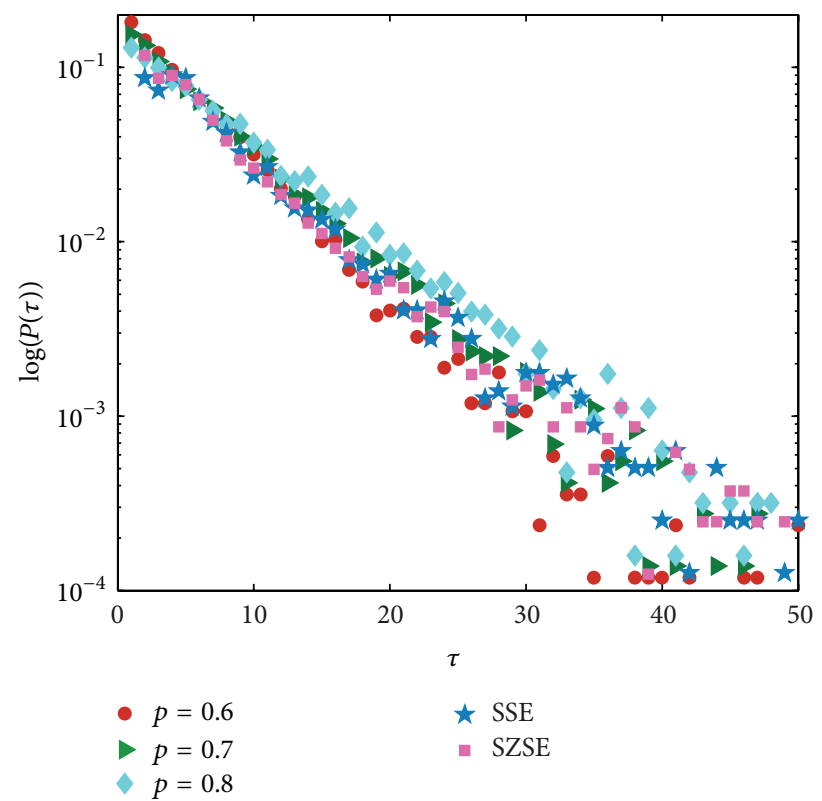

(d)

Figure 6: PDFs of the return intervals on the same threshold $q$ in a semilog plot. (a), (b), (c), and (d) Plots of function $P_{q}(\tau)$ for $q=0.5,1,1.5$, and 2 , respectively.

Composite Index. In order to obviate the effect of the daily vibration, the intraday pattern is scaled and defined by

$$
r^{\prime}(t)=\frac{|r(t)|}{A(s)}=\frac{\left|r^{k}(s)\right|}{A(s)}
$$

where $r(t)=r^{k}(s)$ depends on $k$ and $s$. That is, for any $r(t)$ at time $t$, a corresponding $r^{k}(s)$ at time $s$ in $k$ th trading day exists, and the time scale here is $5 \mathrm{~min}$. The normalized volatility $C(t)$ is defined by

$$
C(t)=\frac{r^{\prime}(t)}{\sqrt{E\left[r^{\prime}(t)\right]^{2}-\left(E\left[r^{\prime}(t)\right]\right)^{2}}} .
$$

The return interval $\tau$ is the random time length between successive volatilities for serial volatility $C(t)$ which depends on the threshold $q$. Figure 5(b) well illustrates the return intervals for SSE on different thresholds in four trading days. As shown in the figure, every volatility $C(t)$ above a threshold $q$ is picked, and the series of the time intervals between those events $\tau(q)$ is generated. In this paper, we mainly investigate the return intervals when the values of $q$ are selected as $0.5,1,1.5$, and 2 , respectively. Recent research shows that the probability density function of the return intervals $P_{q}(\tau)$ follows the scaling relation $P_{q}(\tau)=\langle\tau\rangle^{-1} f(\tau /\langle\tau\rangle)$, where $\langle\tau\rangle$ is the mean return interval and $f(x) \sim e^{-\alpha x^{\gamma}}$; see [31]. In the present paper, we find that the corresponding PDFs of return 


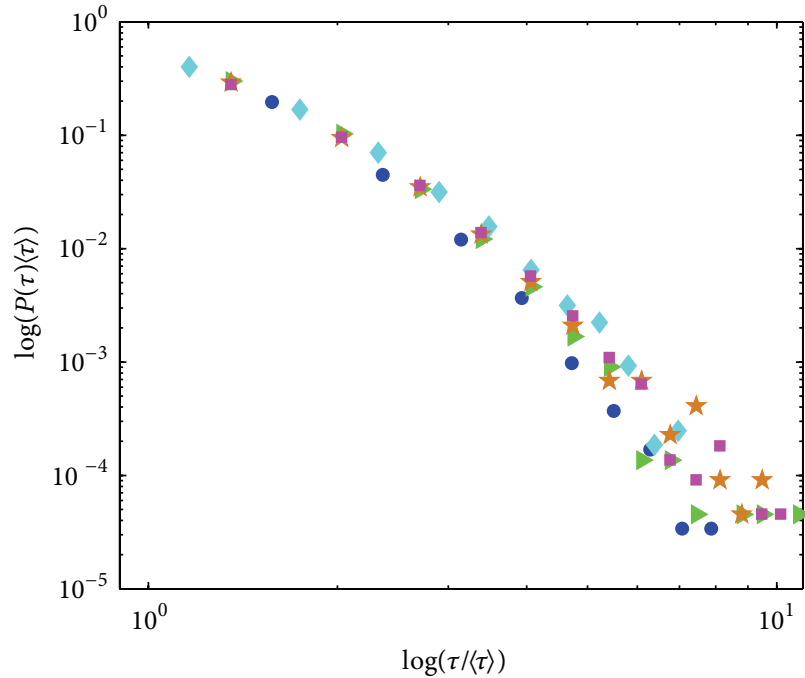

(a)

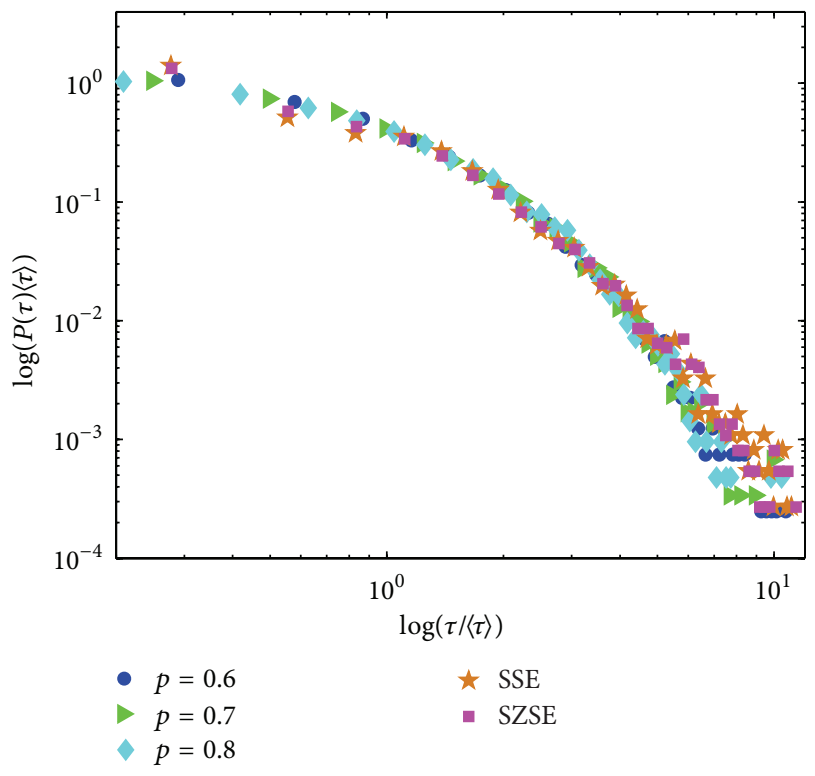

(c)

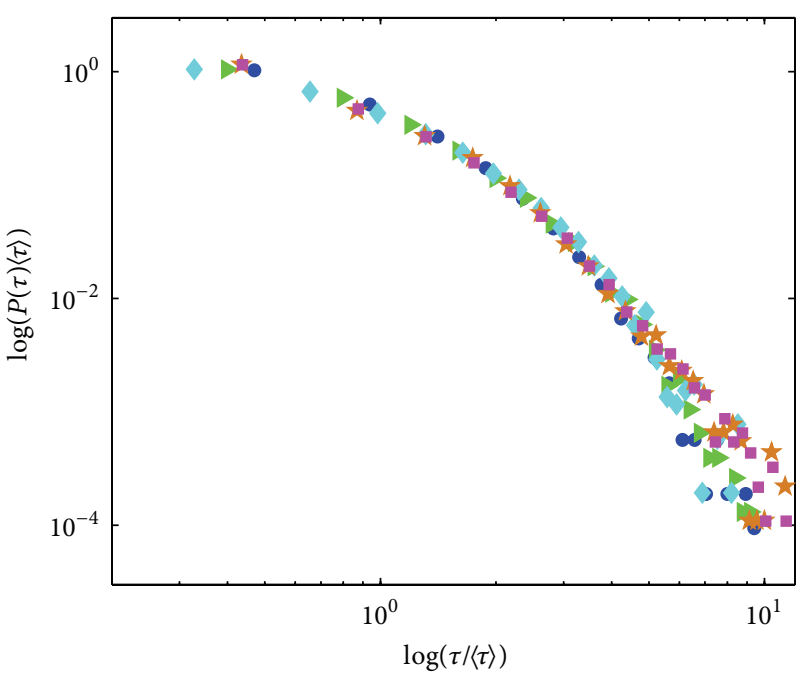

(b)

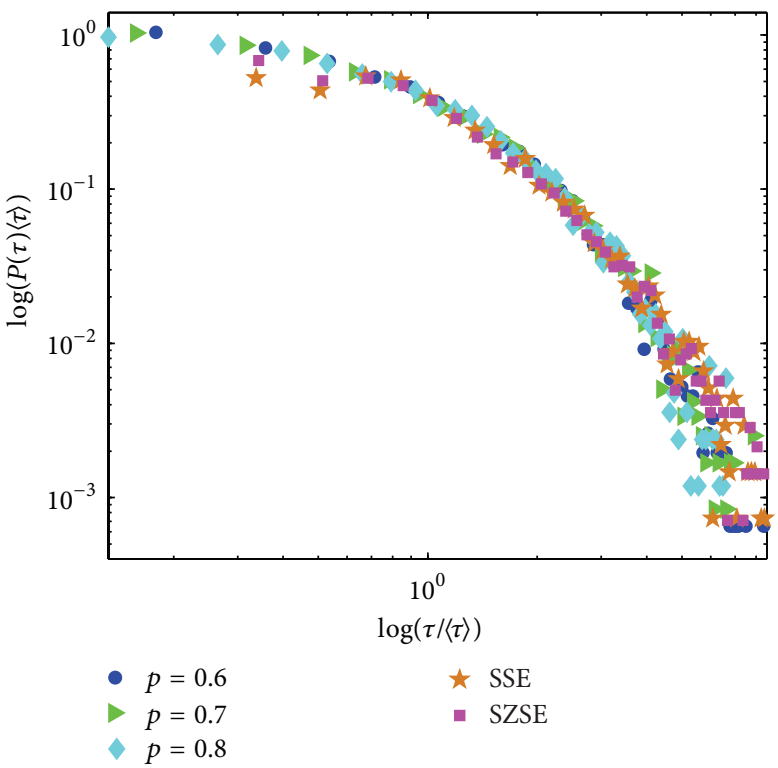

(d)

Figure 7: The scaled probability density functions of return intervals on the same threshold $q$ in a $\log -\log$ plot. (a), (b), (c), and (d) Plots of $P_{q}(\tau)\langle\tau\rangle$ versus $\tau /\langle\tau\rangle$ for $q=0.5,1,1.5$, and 2 , respectively.

intervals for the price model and SSE follow this scaling form, and some new scaling functions are well approximated by other stretched exponential, such as $h(x) \sim e^{-\alpha(\ln x)^{\gamma}}$.

\subsection{Comparisons of Statistical Properties for the Return Inter-} vals. For four fixed threshold values $q$, the probability density functions $P_{q}(\tau)$ of return intervals $\tau$ for the simulation data and SSE, SZSE are given in Figure 6. For the financial model with the parameter values $p$ of $0.6,0.7$, and 0.8 , the number of simulation data is about 48000 , respectively. The actual $5 \mathrm{~min}$ price data from SSE and SZSE indexes have the same quantity.

It can be seen that the distributions of the actual data and the simulation data have the similar trend; especially, when the parameter $p$ ranges from 0.7 to 0.8 , the probability density distributions of the simulation data becomes closer to those of the actual data. Thus, for the discussion in next section, we select the data with $p=0.75$ to stand for the simulation data. Taking a close look at the figure, for fixed return interval $\tau, P(\tau)$ becomes larger with $p$ increasing, which implies that investment information spreading more widely in stock market will cause larger price fluctuations. Further, we study the patterns of function $P_{q}(\tau)\langle\tau\rangle$ versus $\tau /\langle\tau\rangle$ for SSE, SZSE, and the simulation data in Figure 7, and find that the scaled probability density curves of all the data which decay as a power-law line are similar to each other.

4.3. New Scaling Relations of Return Internals for Different Exponent Values. In this section, a scaling function of 


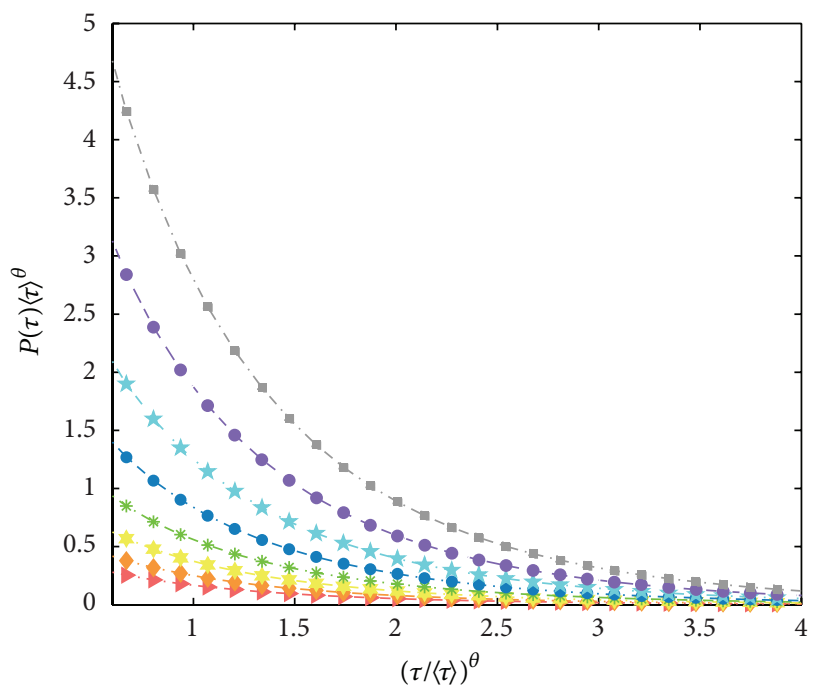

(a)

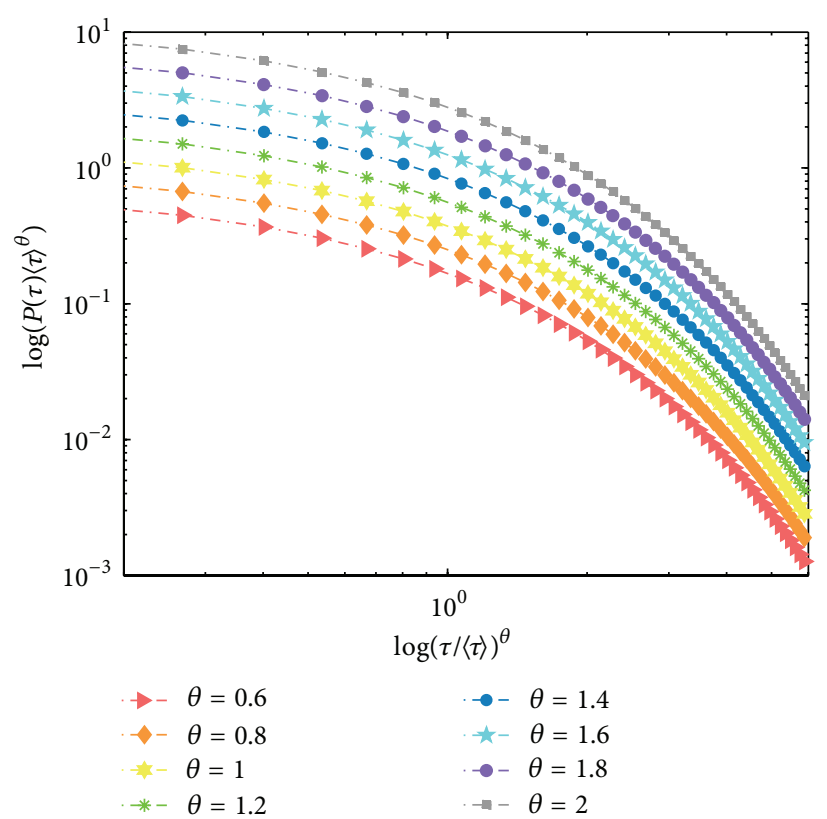

(c)

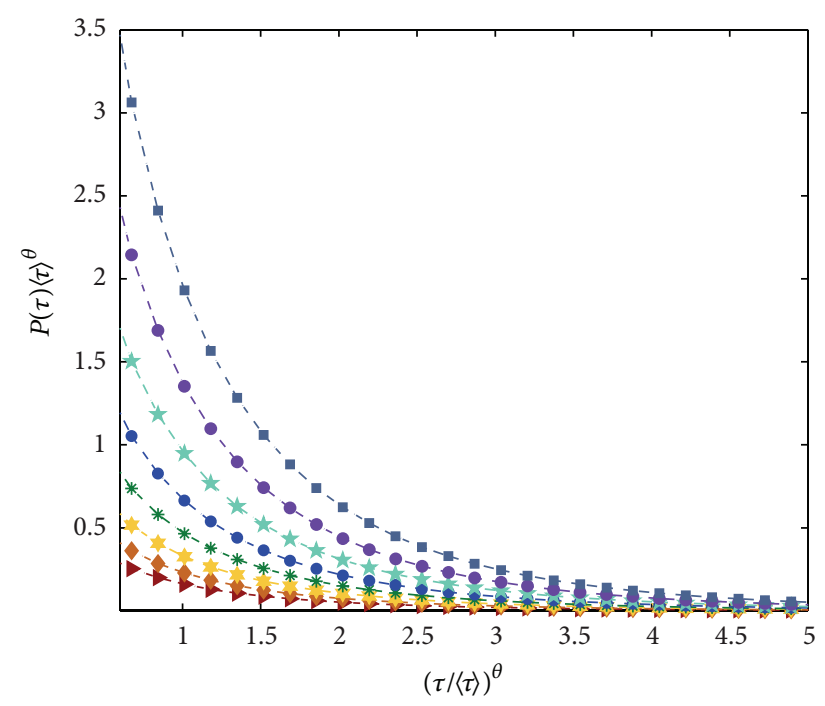

(b)

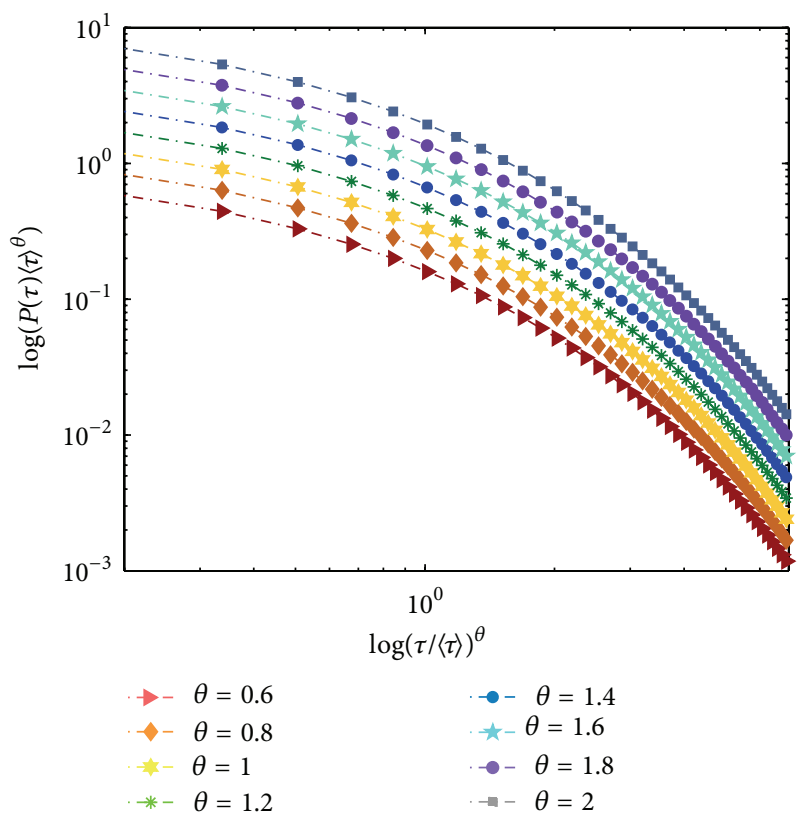

(d)

FIGURE 8: Scaling probability density functions $P(\tau)\langle\tau\rangle^{\theta}$ of return intervals for different vlaues $\theta$ on threshold $q=2$. (a) and (c) Plot and $\log -\log$ plot of the price model with $p=0.75$, respectively. (b) and (d) Plot and log-log plot for SSE, respectively.

exponential parameter is introduced to analyze fluctuation behaviors of return intervals, and this exponent parameter $\theta$ can bring some regular patterns in the present financial research. The scaling relations we will investigate are $P(\tau)\langle\tau\rangle^{\theta}$ versus $(\tau /\langle\tau\rangle)^{\theta}$ and $(P(\tau)\langle\tau\rangle)^{\theta}$ versus $(\tau /\langle\tau\rangle)^{\theta}$. Considering that the length of return intervals series should be rational, we set the threshold $q=2$. The plots of the scaling relation of $P(\tau)\langle\tau\rangle^{\theta}$ versus $(\tau /\langle\tau\rangle)^{\theta}$ are exhibited in Figure 8, where the exponent parameter $\theta$ ranges from 0.6 to 2 with the step of 0.2. Here, all the fitting curves of $P(\tau)\langle\tau\rangle^{\theta}$ are got by the stretched exponential function: $h(x)=\omega e^{-\alpha(\ln x)^{\gamma}}$.

In the following, we investigate the patterns of scaling function $(P(\tau)\langle\tau\rangle)^{\theta}$ versus $(\tau /\langle\tau\rangle)^{\theta}$ for the simulation data and SSE. In Figures 9(a) and 9(c), for different exponent values of $\theta$, the scatterplots show the similar trend, while the patterns of $(P(\tau)\langle\tau\rangle)^{\theta}$ with the exponent $\theta$ increasing can be observed clearly from the three-dimensional view in Figures 9(b) and 9(d). The empirical research shows that 


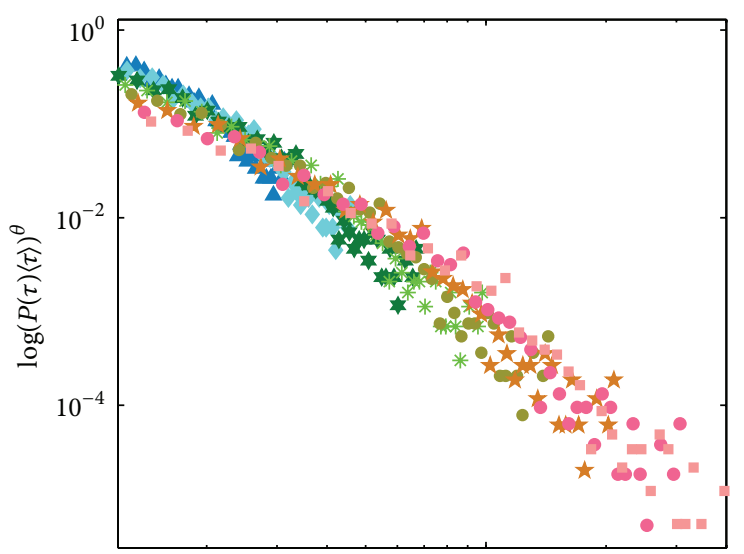

$10^{1}$

$\log (\tau /\langle\tau\rangle)^{\theta}$
$\Delta \theta=0.6$
$\begin{aligned} \theta & =0.8\end{aligned}$

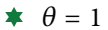
* $\theta=1.2$

(a)

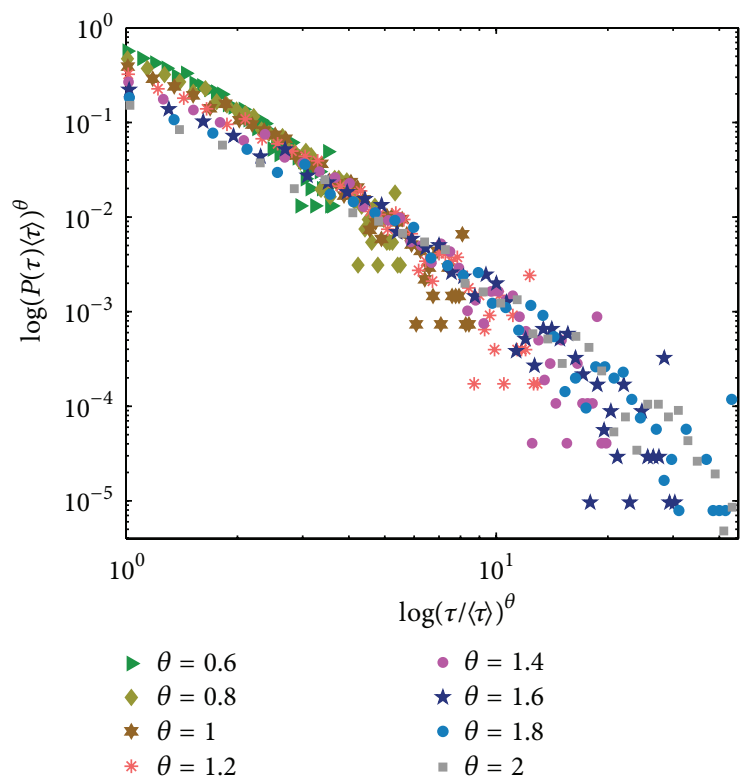

(c)

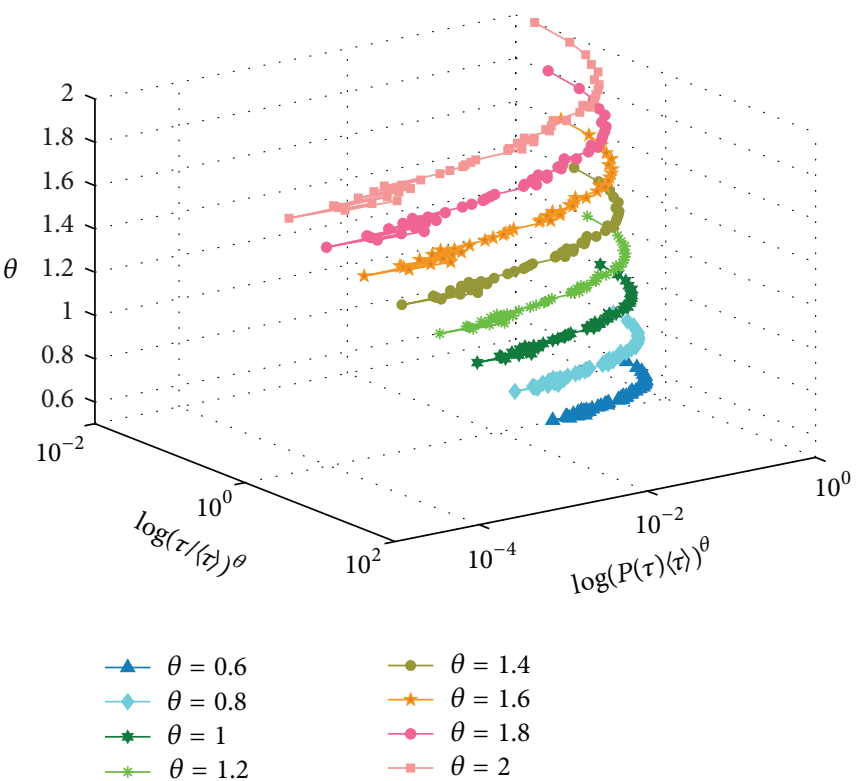

(b)

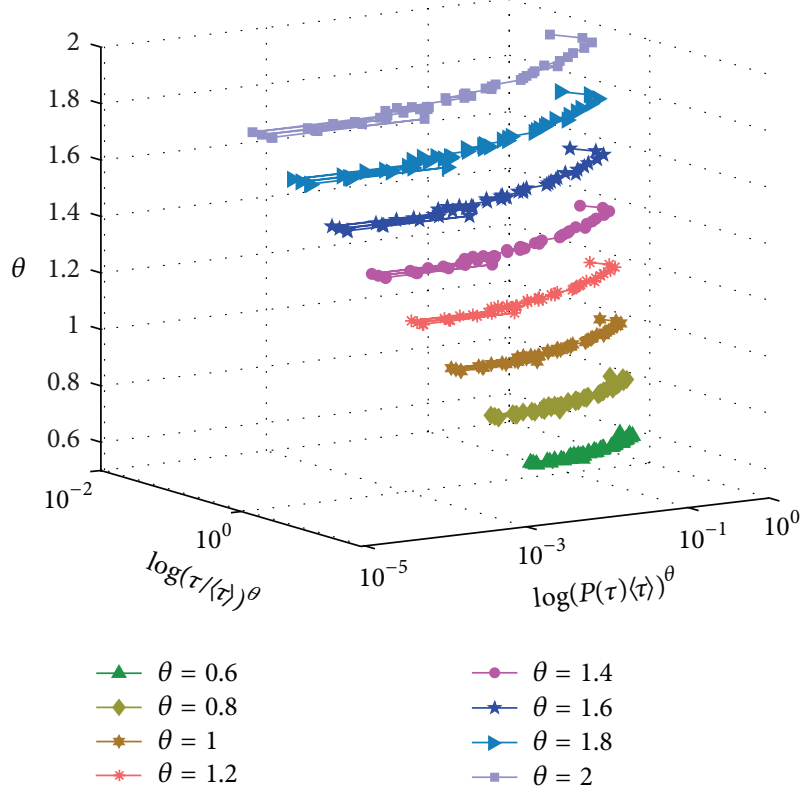

(d)

FIGURE 9: Scaling probability density functions $(P(\tau)\langle\tau\rangle)^{\theta}$ of return intervals for different values $\theta$ on threshold $q=2$. (a) and (b) log-log plot and three-dimensional plot of the price model with $p=0.75$, respectively. (c) and (d) log-log plot and three-dimensional plot for SSE, respectively.

the simulation data and the actual data have the similar scaling form expressed by $(P(\tau)\langle\tau\rangle)^{\theta}=h\left((\tau /\langle\tau\rangle)^{\theta}\right)$, which can be well approximated by the stretch exponential $h(x)=$ $\omega e^{-\alpha(\ln x)^{\gamma}}$. Table 1 shows the statistical analysis of $h(x)$ for the simulation data and SSE index, and all parameters are estimated by the least squares fitting technique. We can find that the fitting curves of the simulation data show the similar behaviors with SSE index; especially, for one database, the values of $\gamma$ keep the same with the various values of $\theta$.

Next we study the distribution of the scaling function $(P(\tau)\langle\tau\rangle)^{\theta}$ with a fixed $\theta$ under different $q$. Firstly, for $\theta=0.9$, we compare the PDF of $P(\tau)$ and the scaling function $(P(\tau)\langle\tau\rangle)^{\theta}$ of return intervals. Figures 10(a) and 10 (c) indicate that the patterns of $P(\tau)$ for four different values 


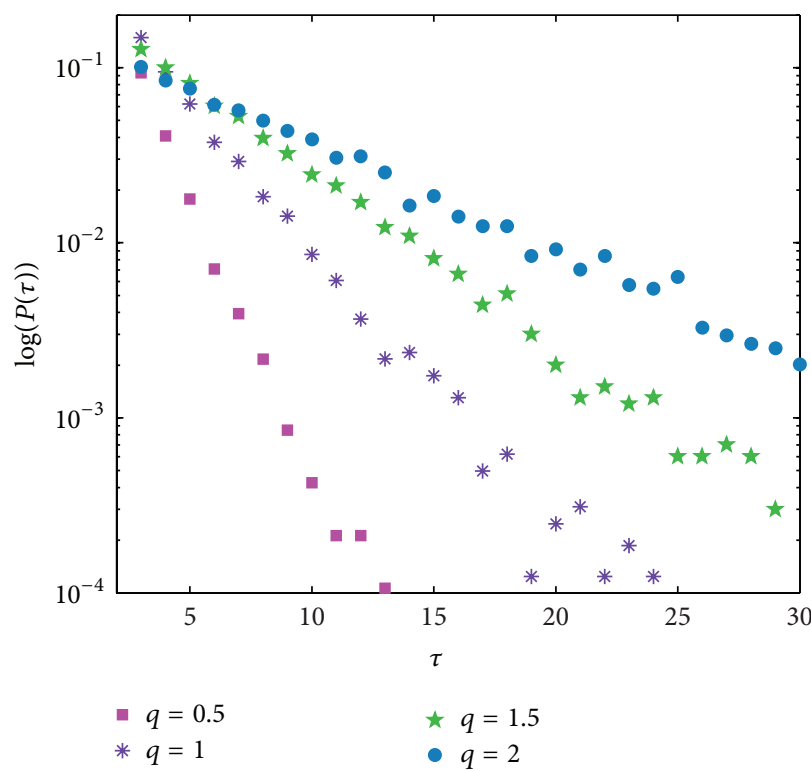

(a)

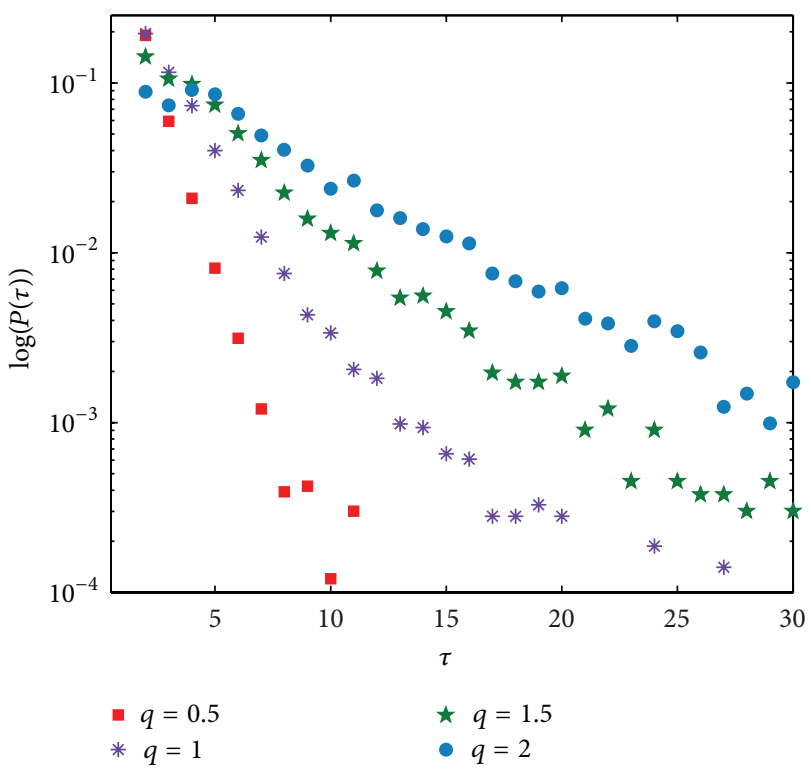

(c)

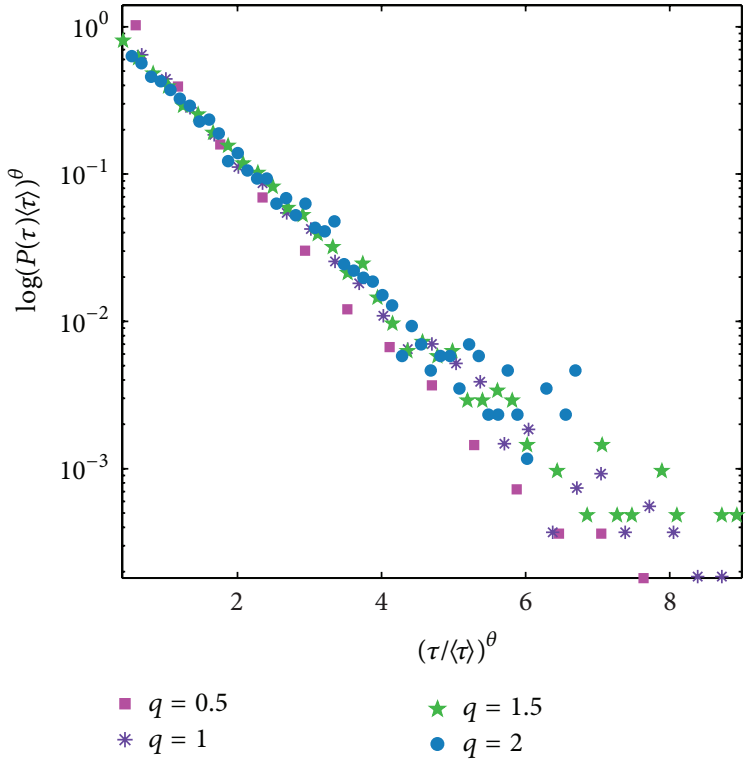

(b)

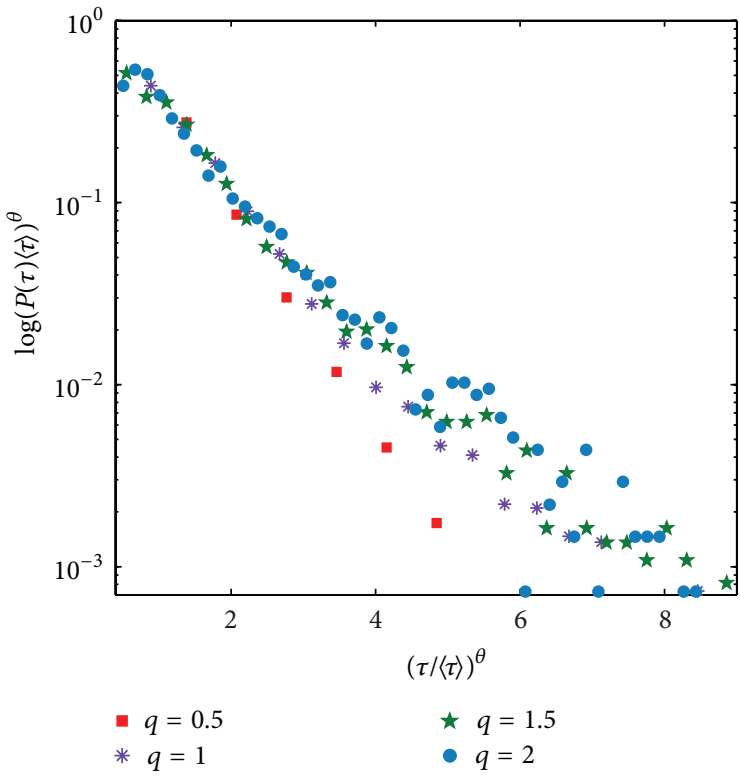

(d)

FIGURE 10: Semilog plots of probability density functions and scaling functions of return intervals for different threshold $q$. (a) and (b) Corresponding plots for the price model. (c) and (d) Corresponding plots for SSE index.

TABLE 1: Statistical analysis of $h(x)$ for the simulation data and SSE.

\begin{tabular}{|c|c|c|c|c|c|c|}
\hline \multirow{2}{*}{$\theta$} & \multicolumn{2}{|c|}{$\omega$} & \multicolumn{2}{|c|}{$\alpha$} & \multicolumn{2}{|c|}{$\gamma$} \\
\hline & S. data & SSE & S. data & SSE & S. data & SSE \\
\hline 0.6 & $0.57 \pm 0.02$ & $0.52 \pm 0.02$ & $1.18 \pm 0.06$ & $1.06 \pm 0.06$ & $1.6 \pm 0.05$ & $1.56 \pm 0.05$ \\
\hline 0.8 & $0.47 \pm 0.02$ & $0.41 \pm 0.02$ & $1.57 \pm 0.06$ & $1.41 \pm 0.06$ & $1.6 \pm 0.05$ & $1.56 \pm 0.05$ \\
\hline 1.0 & $0.39 \pm 0.02$ & $0.33 \pm 0.02$ & $1.96 \pm 0.06$ & $1.76 \pm 0.06$ & $1.6 \pm 0.05$ & $1.56 \pm 0.05$ \\
\hline 1.2 & $0.33 \pm 0.02$ & $0.26 \pm 0.02$ & $2.35 \pm 0.06$ & $2.12 \pm 0.06$ & $1.6 \pm 0.05$ & $1.56 \pm 0.05$ \\
\hline 1.4 & $0.27 \pm 0.02$ & $0.21 \pm 0.02$ & $2.74 \pm 0.06$ & $2.47 \pm 0.06$ & $1.6 \pm 0.05$ & $1.56 \pm 0.05$ \\
\hline 1.6 & $0.23 \pm 0.02$ & $0.17 \pm 0.02$ & $3.13 \pm 0.06$ & $2.82 \pm 0.06$ & $1.6 \pm 0.05$ & $1.56 \pm 0.05$ \\
\hline 1.8 & $0.19 \pm 0.02$ & $0.14 \pm 0.02$ & $3.52 \pm 0.06$ & $3.18 \pm 0.06$ & $1.6 \pm 0.05$ & $1.56 \pm 0.05$ \\
\hline 2.0 & $0.16 \pm 0.02$ & $0.11 \pm 0.02$ & $3.92 \pm 0.06$ & $3.53 \pm 0.06$ & $1.6 \pm 0.05$ & $1.56 \pm 0.05$ \\
\hline
\end{tabular}

Note: S. data means the simulation data. 


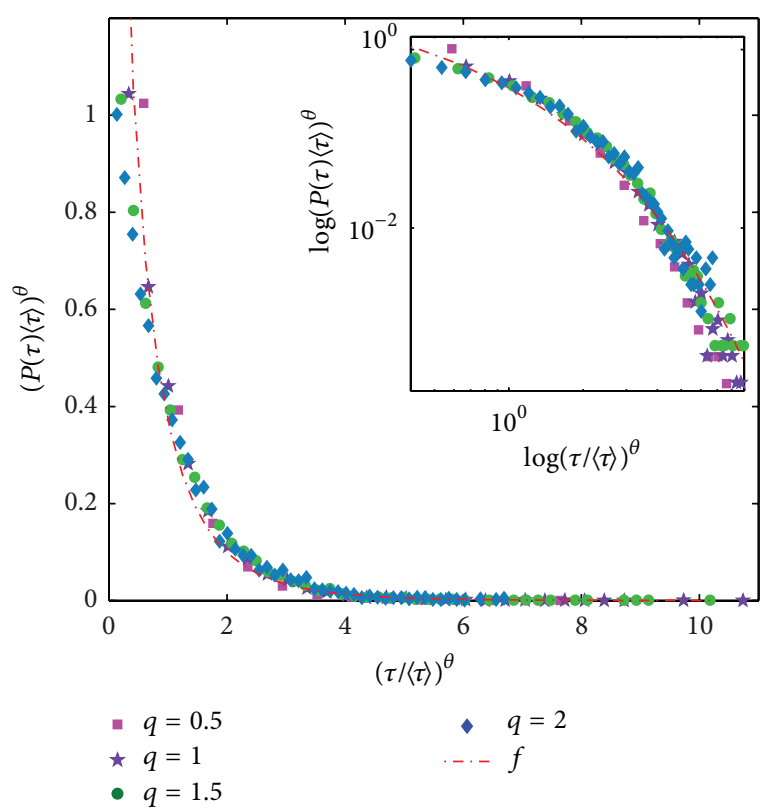

(a)

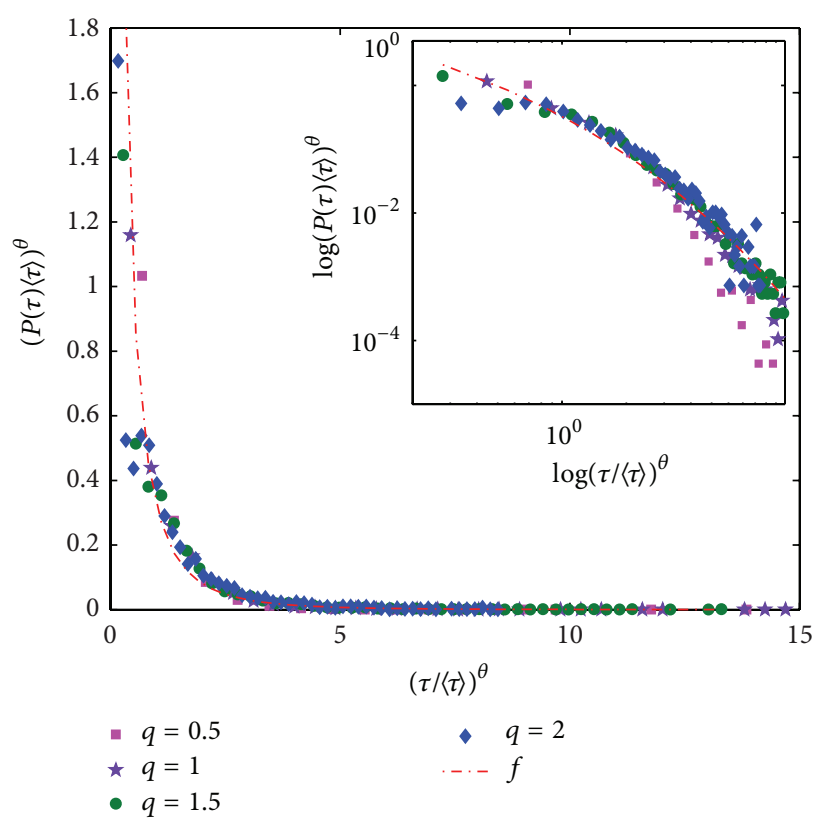

(b)

FIGURE 11: Scaling probability density functions of return intervals for the model and SSE with different threshold $q$. The inset plots are log-log plots and the red lines are the fitted curves. (a) Corresponding plots for the price model. (b) Corresponding plots for SSE index.

of $q$ are separated from each other, which all collapse with $\tau$ increasing, whereas Figures 10(b) and 10(d) exhibit that four patterns of $(P(\tau)\langle\tau\rangle)^{\theta}$ are very close to each other and decay as a line approximately in the semilog plot. From the comparison, it is also found that the distribution of return intervals from the simulation data and SSE has the similar scaling behaviors.

We analyze the scaling function $(P(\tau)\langle\tau\rangle)^{\theta}$ of the model and SSE in detail with $\theta=0.9$ for different $q$. We find that they have similar statistical properties and all databases correspond with a single scaling relation by observing Figure 11. It also supports the view that the scaling relation can be approximated by $h(x)$ which is mentioned in the last paragraph of Section 4.1.

\section{Conclusion}

In the present paper, the oriented percolation is applied to develop a stock price process. We study the statistical properties of the price returns and also investigate the scaling behaviors of the return intervals for the simulation data and the actual data. And a scaling probability density function of exponential parameter is introduced to analyze fluctuation behaviors of return intervals. For different exponential values of $\theta$ and threshold values of $q$, we make statistical analysis of scaled probability density functions for both the simulation data and the actual data. The empirical research exhibits that they have the similar scaling behaviors, and the scaling form can be approximately fitted by a stretched exponential function. All the comparisons reflect that, for the proper parameters, the simulation data of the model can fit the real market to a certain extent.

\section{Acknowledgment}

The authors were supported in part by the National Natural Science Foundation of China Grant no. 71271026 and Grant no. 10971010.

\section{References}

[1] F. Black, "Studies of stock price volatility changes," in Proceedings of the Meetings of the American Statistical Association, Business and Economical Statistics Section, pp. 177-181, 1976.

[2] J. P. Bouchaud, A. Matacz, and M. Potters, "Leverage effect in financial markets: the retarded volatility model," Physical Review Letters, vol. 87, no. 22, Article ID 228701, 4 pages, 2001.

[3] J. P. Bouchaud and M. Potters, Theory of Financial Risks: From Statistical Physics to Risk Management, Cambridge University Press, 2000.

[4] J. Y. Campbell and L. Hentschel, "No news is good news: an asymmetric model of changing volatility in stock returns," Journal of Financial Economics, vol. 31, no. 3, pp. 281-318, 1992.

[5] J. Y. Campbell, A. W. Lo, and A. C. McKinley, The Econometrics of Financial Markets, Princeton University Press, Princeton, NJ, USA, 1997.

[6] R. Cont and J.-P. Bouchaud, "Herd behavior and aggregate fluctuations in financial markets," Macroeconomic Dynamics, vol. 4, no. 2, pp. 170-196, 2000.

[7] Z. Ding, C. W. J. Granger, and R. F. Engle, "A long memory property of stock market returns and a new model," Journal of Empirical Finance, vol. 1, no. 1, pp. 83-106, 1993. 
[8] P. Grau-Carles, "Long-range power-law correlations in stock returns," Physica A, vol. 299, no. 3-4, pp. 521-527, 2001.

[9] Y. L. Guo and J. Wang, "Simulation and statistical analysis of market return fluctuation by Zipf method," Mathematical Problems in Engineering, vol. 2011, Article ID 253523, 14 pages, 2011.

[10] R. A. Haugen, E. Talmor, and W. N. Torous, "The effect of volatility changes on the level of stock prices and expected future returns," The Journal of Finance, vol. 46, no. 3, pp. 9851007, 1991.

[11] Z. Liao and J. Wang, "Forecasting model of global stock index by stochastic time effective neural network," Expert Systems with Applications, vol. 37, no. 1, pp. 834-841, 2010.

[12] L. Sabatelli, S. Keating, J. Dudley, and P. Richmond, "Waiting time distributions in financial markets," The European Physical Journal B, vol. 27, no. 2, pp. 273-275, 2002.

[13] H. E. Stanley, J. S. Andrade Jr., S. Havlin, H. A. Makse, and B. Suki, "Percolation phenomena: a broad-brush introduction with some recent applications to porous media, liquid water, and city growth," Physica A, vol. 266, no. 1-4, pp. 5-16, 1999.

[14] D. Stauffer and A. Aharony, Introduction to Percolation Theory, Taylor \& Francis, London, UK, 2001.

[15] D. Stauffer and T. J. P. Penna, "Crossover in the Cont-Bouchaud percolation model for market fluctuations," Physica A, vol. 256, no. 1-2, pp. 284-290, 1998.

[16] F. Wang and J. Wang, "Statistical analysis and forecasting of return interval for SSE and model by lattice percolation system and neural network," Computers \& Industrial Engineering, vol. 62, no. 1, pp. 198-205, 2012.

[17] J. Wang, Q. Y. Wang, and J. G. Shao, "Fluctuations of stock price model by statistical physics systems," Mathematical and Computer Modelling, vol. 51, no. 5-6, pp. 431-440, 2010.

[18] T. S. Wang, J. Wang, and B. Fan, "Statistical analysis by statistical physics model for the stock markets," International Journal of Modern Physics C, vol. 20, no. 10, pp. 1547-1562, 2009.

[19] X. Wang and J. Wang, "Statistical behavior of a financial model by lattice fractal Sierpinski carpet percolation," Journal of Applied Mathematics, vol. 2012, Article ID 735068, 12 pages, 2012.

[20] D. Xiao and J. Wang, "Modelling stock price dynamics by continuum percolation system and relevant complex systems analysis," Physica A, vol. 391, no. 20, pp. 4827-4838, 2012.

[21] Y. Yu and J. Wang, "Lattice-oriented percolation system applied to volatility behavior of stock market," Journal of Applied Statistics, vol. 39, no. 4, pp. 785-797, 2012.

[22] J. H. Zhang, J. Wang, and J. Shao, "Finite-range contact process on the market return intervals distributions," Advances in Complex Systems, vol. 13, no. 5, pp. 643-657, 2010.

[23] R. Gaylord and P. Wellin, Computer Simulations with Mathematica: Explorations in the Physical, Biological and Social Science, Springer, New York, NY, USA, 1995.

[24] K. Ilinski, Physics of Finance: Gauge Modeling in NonEquilibrium Pricing, John Wiley, New York, NY, USA, 2001.

[25] R. N. Mantegna and H. E. Stanley, An Introduction to Econophysics, Cambridge University Press, Cambridge, UK, 1999.

[26] R. Durrett, Lecture Notes on Particle Systems and Percolation, Wadsworth \& Brooks, California, Calif, USA, 1988.

[27] R. Durrett, "Oriented percolation in two dimensions," The Annals of Probability, vol. 12, no. 4, pp. 999-1040, 1984.

[28] G. Grimmett, Percolation, Springer, Berlin, Germany, 2nd edition, 1999.
[29] T. M. Liggett, Stochastic Interacting Systems: Contact, Voter and Exclusion Processes, Springer, New York, NY, USA, 1999.

[30] J. Wang, J. G. Shao, and B. T. Wang, "Analysis of two-layered random interfaces for two dimensional Widom-Rowlinson's model," Abstract and Applied Analysis, vol. 2011, Article ID 858725, 21 pages, 2011.

[31] F. Wang, K. Yamasaki, S. Havlin, and H. E. Stanley, "Scaling and memory of intraday volatility return intervals in stock markets," Physical Review E, vol. 73, no. 2, Article ID 026117, 8 pages, 2006.

[32] D. Lamberton and B. Lapeyre, Introduction to Stochastic Calculus Applied to Finance, Chapman and Hall/CRC, London, UK, 2000.

[33] S. M. Ross, An Introduction to Mathematical Finance, Cambridge University Press, Cambridge, UK, 1999. 


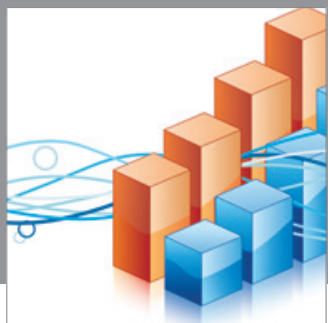

Advances in

Operations Research

mansans

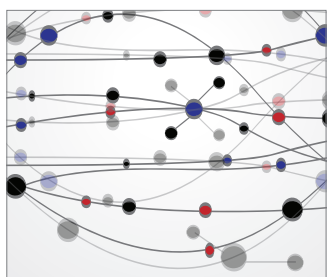

The Scientific World Journal
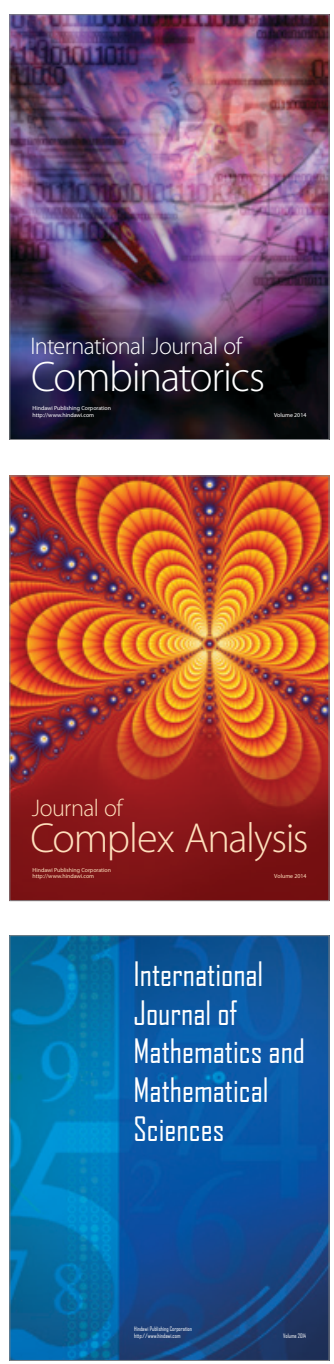
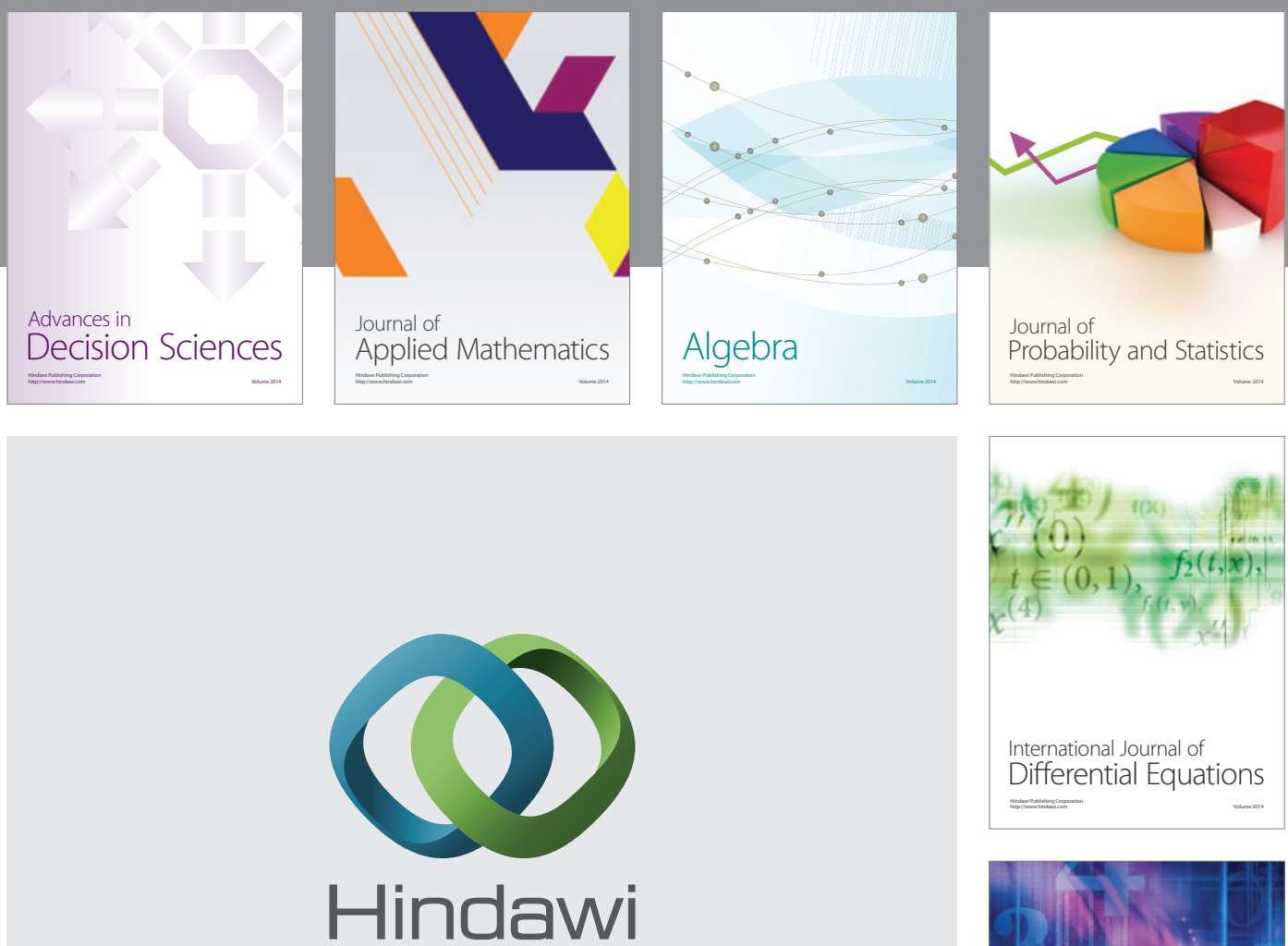

Submit your manuscripts at http://www.hindawi.com
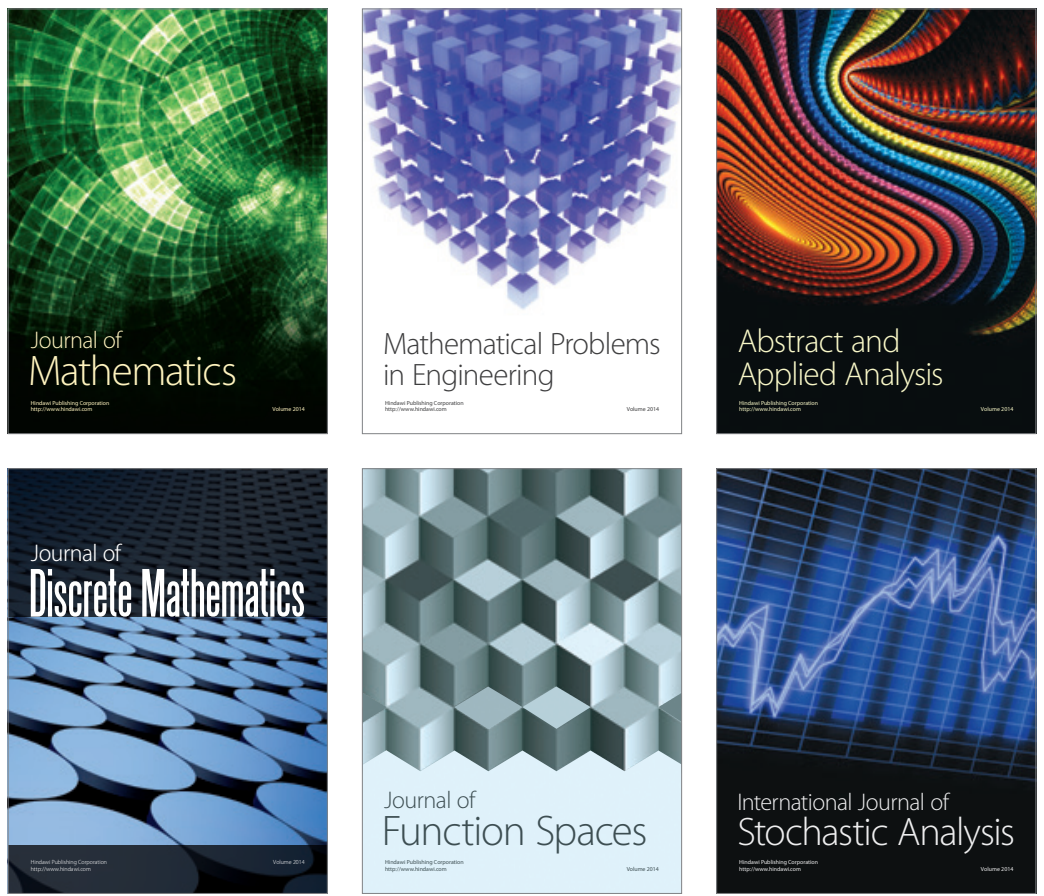

Journal of

Function Spaces

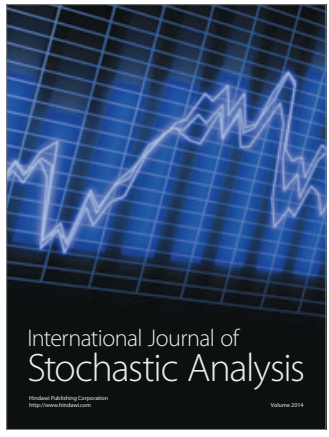

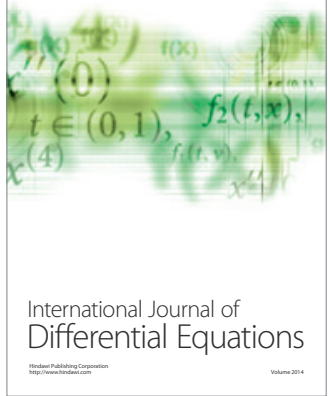
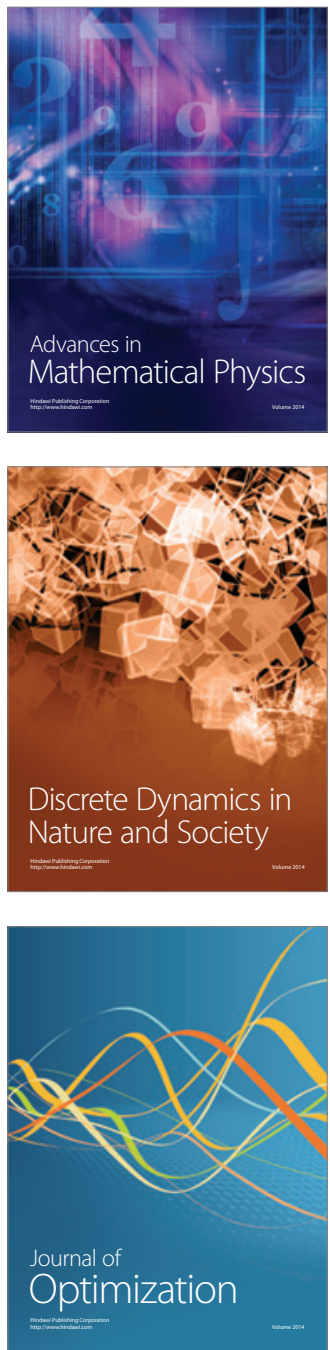